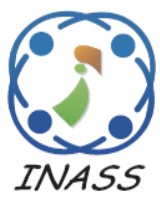

\title{
Accurate DOA Estimation Using Modified ESPRIT Algorithm
}

\author{
Azad Raheem Kareem ${ }^{1 *} \quad$ Ali Majeed Mahmood ${ }^{1}$ \\ ${ }^{I}$ Control and Systems Engineering Department, University of Technology- Iraq, Iraq \\ * Corresponding author's Email: Azad.R.Kareem@uotechnology.edu.iq
}

\begin{abstract}
Direction of Arrival (DOA) estimation represents one of the key issues in the field of signal processing for the present and future 5G and radar engineering applications. The problem of the inaccuracy of DOA estimation has attracted considerable interest from academia. One of the advantageous common techniques that have been followed to improve the accuracy of DOA is the Estimation of the Signal Parameters via the Rotational Invariance Technique (ESPRIT) algorithm, albeit ESPRIT suffers from the challenge of estimation bias at short separation distances when two sources are very close to each other. Hence, in this paper, a modified-ESPRIT algorithm is proposed to improve the accuracy of the DOA. The modification of ESPRIT is presented by increasing the phase difference between the received signals. The idea is to add a pre-processing before the frame of the ESPRIT algorithm; this is done by introducing a double-balanced mixer and $90^{\circ}$ signal splitter at the output of each antenna. The performance evaluation shows that using only the ESPRIT algorithm exhibits large estimation bias when two sources are very close to each other, while the performance is improved noticeably with the proposed modified-ESPRIT. It can detect and resolve two sources with an enhancement of $83.09 \%$ over traditional ESPRIT when the number of snapshots is very short, while an enhancement of $54.4 \%$ is achieved when source separation between (1 to 4 ) degrees and $77.8 \%$ achieved when Signal to Noise Ratio (SNR) between 0-10 dB and of 79.04\% when four or fewer elements are used. The obtained results validate the performance efficiency of the proposed algorithm in terms of less Root Mean Square Error (RMSE), and a smaller number of snapshots.
\end{abstract}

Keywords: ESPRIT, DOA estimation, Eigenvector method, Multiple signal classification, Principal component analysis.

\section{Introduction}

All Direction-of-Arrival (DOA) estimation has an important role in radar, sonar, mobile networks, remote sensing, and other communication applications [1, 2]. The Sensor-based technology such as Lidar, vision, inertial measurement unit, and sonar can only deliver local position estimation, while in the signal-based technology like $\mathrm{Wi}-\mathrm{Fi}$ and cellular have no such limitations. Among the different signal-based technologies, cellular LongTerm Evolution (LTE) signals appear to be very attractive due to their geometric diversity, abundance, and wide bandwidth [3, 4].

Several DOA estimators have been used, including the Multiple Signal Classification (MUSIC) [5] and the Estimation of the Signal
Parameters via the Rotational Invariance Technique (ESPRIT) [6-8], algorithms which have been commonly deployed in DOA estimation. ESPRIT method is used for frequency and angle estimation $[6,9]$. The ESPRIT-based joint angle and frequency estimation methods can maintain satisfactory estimation performances, but require additional pairing. The authors in [10] improve ESPRIT algorithm to obtain accurate parameters in short time window, while in [11] the improved ESPRIT algorithm has been used which can estimate efficiently the DOA of the coherent signals Moreover, to reduce the computational complexity, the enhanced ESPRIT, namely the unitary ESPRIT, has been proposed in [12].

Likewise, the Eigenvector Method (EVM) has been applied widely in adaptive signal processors. The former techniques have attracted impressive 
enthusiasm from the academia and researchers since they appear to give a basic way to deal with the DOA estimation issue. It is worth stating that the EVM is similar to MUSIC in producing the results of the spatial spectrum, except that the beam eigenvector is weighted by its eigenvalue [5]. However, the main challenge of the EVM and MUSIC is the computation burden needed in analysis of the full eigenvector and valuation of spatial spectrums through all noise eigenvectors.

ESPRIT was developed to overcome the computation burden, the prior information requirement and the storage costs that have been included in the MUSIC algorithm. ESPRIT uses a signal subspace to estimate the arrival angles of signals with an array geometry, which represents a distinct advantage over the MUSIC algorithm. In contrary to the ESPRIT algorithm, when the MUSIC attempts to estimate arrival angels, it has to search over the set of all possible arrival angles for estimation. Due to the advantages of ESPRIT [13], it has been used broadly in many practical applications, such as radar on-chip and as multiple input multiple output automotive radars [14]. The drawback that has been found in the operation of the ESPRIT algorithm is that the performance of DOA estimation starts to degrade when the space between the sources becomes smaller [15]. It is worth stating that the subspace-based estimators often rely on the statistical properties of the sampled data, thus large numbers of snapshots are required for precision estimation [16].

In this paper, a modified-ESPRIT algorithm is proposed to overcome the problem of the traditional ESPRIT algorithm.

The main contribution is represented by increasing the phase difference between the received signals to minimize the problem of estimation bias at short separation distances in the classical ESPRIT algorithm.

The rest of the paper is structured as follows; in section 2 a detail description of the concept of DOA along with the most common estimation algorithms are illustrated. Section 3 demonstrates the proposed modified-ESPRIT algorithm with its mathematical derivation. Section 4 presents the performance evaluation and discussion for the proposed algorithm. Section 5 shows the conclusions.

\section{Direction of arrival concepts}

Several techniques have been used in directionfinding. The most common one is based on the phase of the signal that progressively varies along its path as shown in Fig. 1.

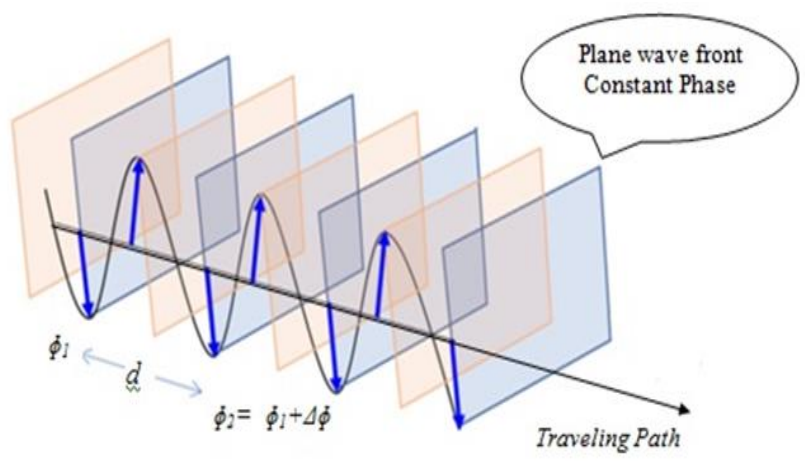

Figure. 1 Phase variation of the plane wave

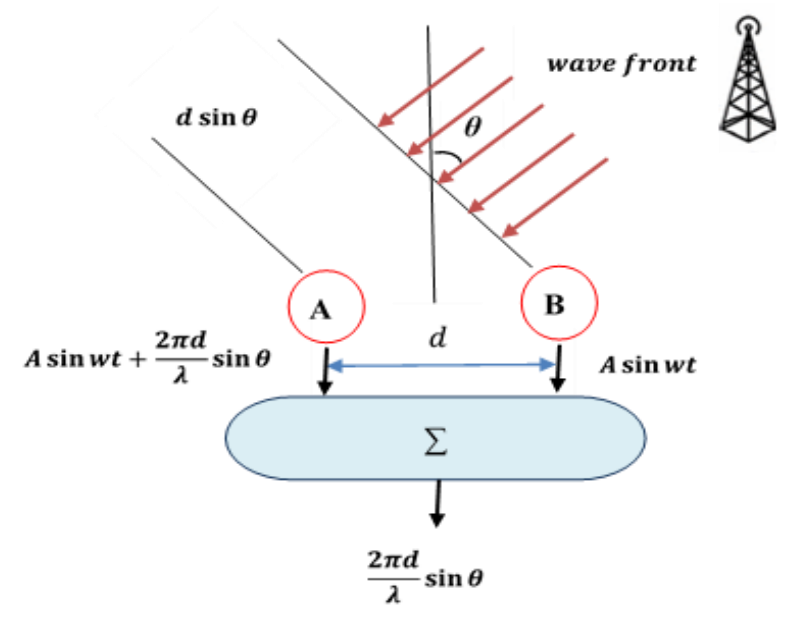

Figure. 2 Direction finding of the plane wave front of the classical ESPRIT

To determine the direction of arrival from time or phase differences by using two antenna- elements $[17,18]$, direction finder antenna array which has to consist of two or more elements that are physically displaced from each other as shown in Fig. 2. [19].

The receivers, A and B as illustrated in Fig. 2 with omnidirectional antennas are arranged on a known baseline which is assumed to be short for the distance to the emitters. Hence, the transmission paths to the two receivers can be considered parallel and then the phase delay is expressed as follows:

$$
\phi=\frac{2 \pi d}{\lambda} \sin \theta=\frac{2 \pi d f}{c} \sin \theta
$$

where, $d$ is the distance between antenna elements, $\lambda$ is the wavelength, $f$ is the frequency, $\mathrm{c}$ is the speed of light, and $\theta$ is the angle of arrival.

The phase front of a plane wave arrives at angle $\theta$ from normal line direction which must travel the additional distance (d). Many studies have been conducted to test the performance of the ESPRIT $[20,21]$. These studies have made a statistical analysis of the subspace-based DOA in the presence of noise with unknown covariance. In the next 
subsections, brief descriptions are demonstrated for three common DOA estimation algorithms.

\subsection{Principal components analysis method}

Principal Component Analysis (PCA) has been used to find the principal component of standardized variables. It has been designed to decrease the dimensionality of the information set comprising of high number of interrelated variables in an interpretable manner, whereas preserving as much as conceivable the variability of the data $[22,23]$. The mathematical representation of this method is expressed in Eqs. (2) and (3) as follows:

$$
\left.\begin{array}{c}
R_{x x}=\sigma^{2} I+\sum_{i=1}^{k} v_{i} e_{i} e_{i}^{H} \\
\text { or } R_{x x}=R_{w w}+R_{s s}
\end{array}\right\}
$$

where, $v$ is the eigenvalues of $R_{x x}, e_{i}$ are the associated eigenvectors of $R_{x x}, R_{x x}$ is the total covariance matrix, $R_{w w}$ is the noise covariance matrix, $R_{S S} i$ s the signal covariance matrix, and $H$ denotes as Hermitian. The $k$ largest eigenvectors in Eq.(2) are called signal subspace, and the $(M-k)$ eigenvector corresponding to the $(M-k)$ smallest eigenvalues they normally have the same value called the noise subspace [24].

Now, it is possible to separate the two covariance matrices mentioned above, the principal component spectra are given by:

$$
P_{P C A}(\theta)=\left[C(\theta) R_{S S}^{-1} C^{H}(\theta)\right]^{-1}
$$

where, $C$ is a search vector.

The spectral estimate represented in Eq. (3) is called the principal components method, since, it uses the principal eigenvectors of the signal subspace [25]. The term signal subspace arises from the fact that this algorithm relies on separating the space spanned by received data into what are called signal and noise subspaces [26].

\subsection{MUSIC method}

MUSIC algorithm was suggested by Schmidt [5] to determine both theoretically and experimentally, some of the important parameters of multiple wavefronts arriving at an antenna array. The parameters include direction of arrivals. The wavefronts that have been received by $M$ array elements are linear combination of $k$ incident wave fronts and noise. Thus, MUSIC begins with the following model:

$$
\left[\begin{array}{c}
x_{1} \\
x_{2} \\
\cdot \\
\cdot \\
x_{M}
\end{array}\right]=\left[\begin{array}{llll}
C\left(\theta_{1}\right) & C\left(\theta_{1}\right) & \cdot & \cdot
\end{array}\right.
$$

or

$$
X=C S+W
$$

The estimated covariance matrix from the collected data is:

Then,

$$
R_{x x}=\mathrm{E}\left[X X^{*}\right]
$$

$$
R_{x x}=C \bar{S} \bar{S}^{*} C^{*}+\bar{W} \bar{W}^{*}
$$

where, * denotes as conjugate, and $E[]$ is the expected values. In special case when $W$ has zero mean and variance of $\sigma^{2}$ then Eq. (6) becomes:

$$
R_{x x}=C \bar{S} \bar{S}^{*} C^{*}+\sigma^{2} I
$$

Now, the $M$ eigenvectors of $R_{x x}$ satisfy:

$$
R_{x x} e_{i}=\sigma^{2} I e_{i}
$$

The eigenvectors associated with minimum eigenvalues are orthogonal to the signal subspace, a subspace spanned by column of $C$.

$$
P_{M U S I C}(\theta)=\frac{1}{C(\theta) E_{N} E_{N}^{H} C^{H}(\theta)}
$$

where, $E_{N}=\left[\begin{array}{llllll}e_{d+1} & e_{d+2} & e_{d+3} & . & . & e_{M}\end{array}\right]$

Finally, it can find $k$ (largest) peak of $P$ to obtain the DOA.

\subsection{Eigenvector method}

The authors in [27, 28] have developed an approach to improve the DOA resolution by using eigenvalue decomposition method. The inverse covariance matrix can be obtained as:

$$
R^{-1}=\sum_{i=1}^{M} v^{-1} e_{i} e_{i}^{H}
$$

The maximum likelihood estimator equation can be rewritten as a function of eigenvalues and eigenvectors as follows to evaluate the spectral beam of only the noise subspace as:

$$
P_{E V M}(\theta)=\frac{1}{\sum_{i=k+1}^{M} v_{i}^{-1}\left|C^{H}(\theta) e_{i}\right|^{2}}
$$


It is worth stating that the eigenvector method is equivalent to MUSIC via assuming that all the noise eigenvalues are equal, excepting that the eigenvector beam are weighted through their eigenvalues. PCA, on the other hand, has its disadvantage at highdimensional conditions when variables number is equal or larger than the number of the observations; it reveals inconsistent estimates leading to unreliable conclusions [29]. In addition, ESPRIT outperforms MUSIC algorithm in terms of computational overhead since it can function without parameter search and iterative calculation. As well as, MUSIC is incapable of resolving two sources in various instances compared to ESPRIT algorithm [30, 31]. However, one of challenges of ESPRIT algorithm is the estimation bias at short separation distances for sources that are very close to each other. Hence, to improve the operation of ESPRIT algorithm, a modification has been introduced to the classical algorithm as clarified in the next sections.

\section{The proposed modified-ESPRIT algorithm}

From the intensive study of the ESPRIT algorithm, there will be a real inaccuracy in the estimation of DOA particularly for small separation angle between sources, where ESPRIT offers a large amount of error. Therefore, this work is directed toward improving the performance of ESPRIT. The idea is to add a pre-processing before the frame of the ESPRIT algorithm so that the basis of ESPRIT foundation perceives no dramatic changes and the benefits of ESPRIT still exist in the modified algorithm. The theory related to this modification is that the maximum separation for a total phase change of no more than $2 \pi$ (for unambiguous angle measurement) is half the wavelength. Eq. (1) shows the errors in the phase measurement are directly proportional to the error in baseline distance $d$. To gain the advantage of baselines longer than $1 / 2$, (increase phase variation), it is possible to use several baselines, with the shorter ones resolving the angular ambiguities of longer lines. The total phase difference between the signals from two antennas is proportional to baseline distance. It is possible to increase the phase difference so that baseline appears to be multiplied in length. the proposed way to do this is to introduce a double-balanced mixer and $90^{\circ}$ signal splitter at the output of each antenna with an array of antennas be composed of two sensors separated by distance $d$ (measured in wavelength), as shown in Fig. 3.

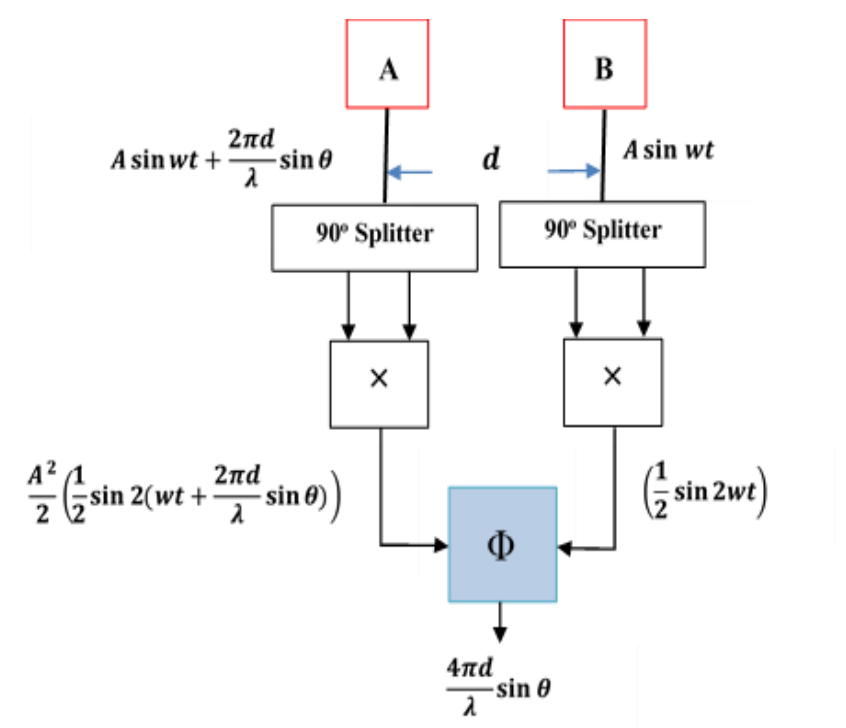

Figure .3 Processing the output of antennas to increase the phase variation of the modified ESPRIT

Fig. 3 demonstrates how the antenna output can be processed to achieve a phase difference which is larger than that due to angel of arrival and the baseline. Referring to classical DOA (Fig. 2.), while in the proposed one as shown in Fig. 3, the signals received by sensors A and B are illustrated by:

$$
\begin{aligned}
& x_{B}(t)=A \sin w t \\
& x_{A}(t)=A \sin \left(w t+\frac{2 \pi d}{\lambda} \sin \theta\right)
\end{aligned}
$$

Then the output of the unity gain phase splitter with gain will be as follows:

$$
\begin{aligned}
x_{B 1}(t) & =A \sin w t \\
x_{B 2}(t) & =A \sin \left(w t+\frac{\pi}{2}\right)=A \cos w t \\
x_{A 1}(t) & =A \sin \left(w t+\frac{2 \pi d}{\lambda} \sin \theta\right) \\
x_{A 2}(t) & =A \sin \left(w t+\frac{2 \pi d}{\lambda} \sin \theta+\frac{\pi}{2}\right) \\
\text { or } \quad x_{A 2}(t) & =A \cos \left(w t+\frac{2 \pi d}{\lambda} \sin \theta\right)
\end{aligned}
$$

From Eqs. (14), (15), (16) and (17), the output of the mixers in the two branches will be as presented in Eqs. (18) and (19).

$$
\begin{aligned}
Y_{B}(t) & =X_{B 1}(t) \cdot X_{B 2}(t)=\frac{A^{2}}{2}\left(\frac{1}{2} \sin 2 w t\right) \\
Y_{A}(t) & =X_{A 1}(t) \cdot X_{A 2}(t) \\
& =\frac{A^{2}}{2}\left(\frac{1}{2} \sin 2\left(w t+\frac{2 \pi d}{\lambda} \sin \theta\right)\right)
\end{aligned}
$$


Finally, after summing Eqs. (18) and (19), the output of the phase detector will be:

$$
\phi=\frac{4 \pi d}{\lambda} \sin \theta
$$

Comparing Eq. (20) with Eq. (1), it can be observed obviously that the phase difference is increased and this increment improves DOA estimation.

\section{Application of the phase difference increment to ESPRIT algorithm}

The phase difference is increased by processing the incoming data using the ESPRIT algorithm to estimate the DOA as shown in Fig.4(a). The proposed model showed in Fig.4(b), is based on preprocessing the incoming data before its processing by ESPRIT.

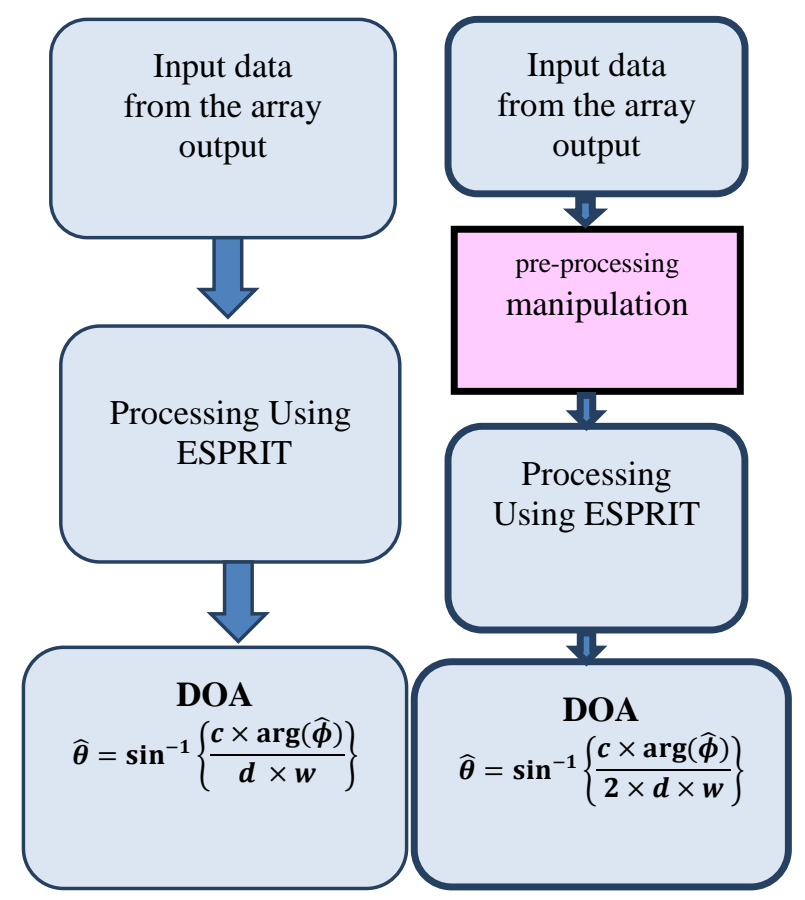

(a)

(b)

Figure. 4 Data processing model of: (a) classical ESPRIT and (b) proposed model

The algorithm of the proposed model is arranged as follows:

\footnotetext{
Algorithm 1: Modified-ESPRIT algorithm

Input = Sources: frequency, amplitude, and angle.

Sensors: number of sensors, the distance between sensors. SNR, number of snapshots and sources.

Output $=$ Direction of arrival estimation.

1: Generate random signal of source signals and compute the covariance

2: Compute the Eigenvalues and the Eigenvectors, then sort them in descending order to estimate signal subspace and noise subspace.

3: Reconstruct the matrix $\mathrm{R}_{\mathrm{xx}}$.
}

4: $\quad$ Obtain the signal subspace by using Singular Value Decomposition (SVD) of $\mathrm{R}_{\mathrm{xx}}$.

5: Partition $\mathrm{E}_{\mathrm{z}}$ into $\mathrm{E}_{\mathrm{x}} \& \mathrm{E}_{\mathrm{y}}$

6: Compute the eigenvalue of $\mathrm{E}_{\mathrm{z}}$.

7: Sort eigenvalue and associated eigenvector of $\mathrm{E}$.

8: Partition $\mathrm{E}$ in to $(\mathrm{d} \times \mathrm{d})$ sub matrices.

9 Compute $\boldsymbol{\Psi}=-\mathbf{E}_{12} \mathbf{E}_{22}{ }^{-1}$.

10 Compute eigenvalue and eigenvector of $\Psi$ which is equal to the diagonal of $\Phi$.

11 Estimate DOA from $\Phi$ according to Eq. (20).

12 End

\section{Performance evaluation and discussion}

The performance of the proposed modifiedESPRIT algorithm is studied deeply, and its behaviour for the different affecting parameters is compared. The comparison is achieved with EVM [32], MUSIC method [33], ESPRIT method [6] and PCA, which consider as subspace-based methods [34]. The comparisons determine how accurate can these methods estimate the DOA, and to which extent the two sources close to each other with a possible resolution. The test based on 300 different independent trials with sinusoids sources in additive white gaussian noise used. The sources frequencies are $0.25 f_{\mathrm{s}}$ and $0.3 \mathrm{f}_{\mathrm{s}}$.

\subsection{Effect of snapshots}

Fig. 6 shows the number of snapshots versus the RMSE of DOA estimation in degree for EVM, MUSIC, ESPRIT, PCA and proposed modifiedESPRIT. The simulation test is achieved for two sources at 5 and 10 degrees, and the snapshots up to 500 snapshots. The modified ESPRIT has better performance than others since it possesses a lower mean square error. The most interesting observation here is that the proposed modified ESPRIT is

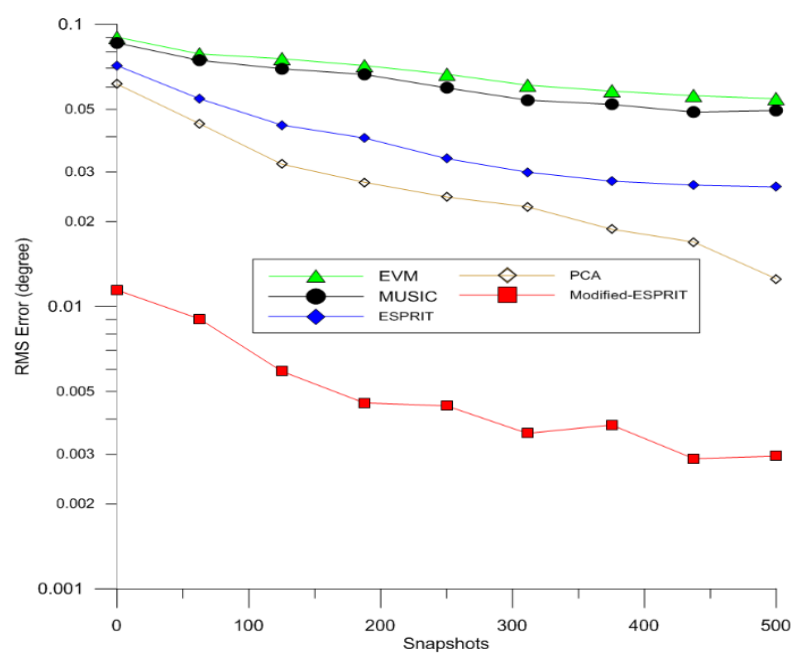

Figure. 6 RMS error vs. the number of snapshots for different DOA methods 
Table 1. Comparison results of the modified-ESPRIT and different methods in terms of the number of snapshots.

\begin{tabular}{|c|c|c|c|}
\hline \multirow[b]{2}{*}{ Algorithm } & \multicolumn{3}{|c|}{$\begin{array}{llll}\begin{array}{l}\text { Root } \\
\times 10^{-2}\end{array} & & \\
\end{array}$} \\
\hline & $\begin{array}{l}\text { Less than } \\
5 \\
\text { snapshots }\end{array}$ & $\begin{array}{l}\text { Between } 5 \text { - } \\
200 \\
\text { snapshots }\end{array}$ & $\begin{array}{l}\text { More } \\
\text { than } 200 \\
\text { to } \quad 500 \\
\text { snapshots }\end{array}$ \\
\hline EVM [28] & 9.0 & 7.5 & 6.0 \\
\hline MUSIC [30] & 8.5 & 7.0 & 5.3 \\
\hline ESPRIT [6] & 7.1 & 4.6 & 3.0 \\
\hline PCA [23] & 6.1 & 3.5 & 2.0 \\
\hline $\begin{array}{l}\text { Modified- } \\
\text { ESPRIT }\end{array}$ & 1.2 & 0.7 & 0.4 \\
\hline
\end{tabular}

capable of estimating the DOA and resolving the two sources when the number of snapshots is very short with a very low standard deviation of fewer than 0.02 degrees, while for the other algorithms, the standard deviation is very large.

The modified-ESPRIT can detect and resolve two sources with an enhancement of $83.09 \%$ over traditional ESPRIT. Here, the RMSE is $0.012^{\circ}$ when the number of snapshots is very short and equal to $0.007^{\circ}$ for snapshots between 5 to 200 , while any of the aforementioned algorithms failed to resolve these two sources correctly, as shown in Table 1.

\subsection{Effect of source separation}

When two sources are sufficiently apart, any estimator is capable of correctly estimating the DOA. However, there is a separate limit for any estimator to be able to resolve these two sources properly and beyond this limit, no resolution can be achieved. Fig.7. shows the estimation of DOA using EVM, MUSIC, ESPRIT, PCA and proposed modifiedESPRIT. When the source separation is 50 degree all estimators have good resolution and can estimate the DOA correctly. But then when the source separation reduced to 10 degrees, ESPRIT resolved the two sources but with a considerable value of estimation bias, while the modified-ESPRIT has no estimation bias. As the source separation reduced to $0.25^{\circ}$ degree ESPRIT and the others are failed to estimate DOA of the two sources accurately although the source separation is within the resolution limit. Hence, the advantage of modifiedESPRIT over classical ESPRIT and other estimators is very clear here. Fig.7 demonstrates that the modified-ESPRIT possesses a minimum standard deviation which means the improvement achieved is very important.

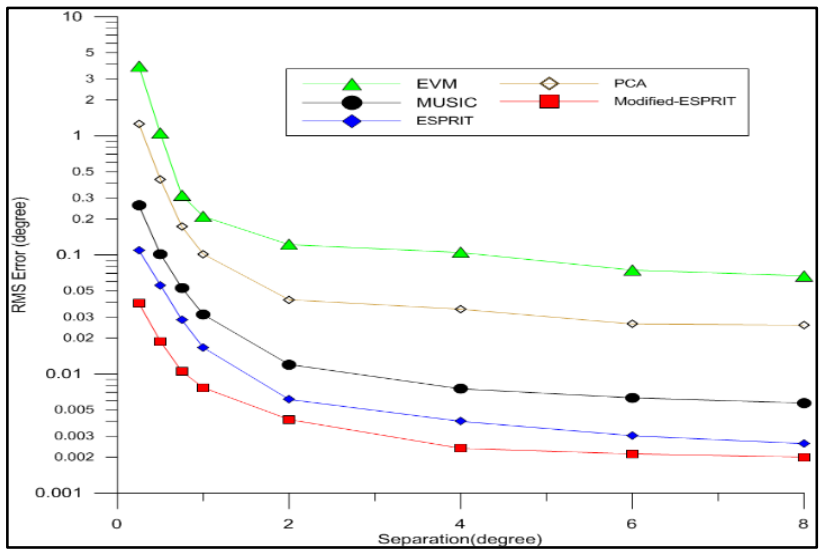

Figure. 7 RMS error vs. source separation for different DOA methods

Table 2. Comparison results of the modified-ESPRIT and different methods in terms of angular resolution

\begin{tabular}{|c|c|c|c|}
\hline \multirow[b]{2}{*}{ Algorithm } & \multicolumn{3}{|c|}{$\begin{array}{l}\text { Root Mean Square Error (deg.) } \\
\times 10^{-2}\end{array}$} \\
\hline & $\begin{array}{l}\text { Less than } \\
\mathbf{1}^{\mathbf{0}}\end{array}$ & $\begin{array}{l}\text { Between } \\
1^{0}-4^{0}\end{array}$ & $\begin{array}{l}\text { More } \\
\text { than } 4^{0} \\
\text { up to } 8^{0}\end{array}$ \\
\hline EVM [28] & 17.3 & 1.66 & 0.82 \\
\hline MUSIC[30] & 1.38 & 0.21 & 0.06 \\
\hline ESPRIT[6] & 0.64 & 0.11 & 0.03 \\
\hline PCA [23] & 6.20 & 0.72 & 0.29 \\
\hline $\begin{array}{l}\text { Modified- } \\
\text { ESPRIT }\end{array}$ & 0.03 & 0.05 & 0.02 \\
\hline
\end{tabular}

Table 2 demonstrates the capability of the modified-ESPRIT algorithm to achieve resolution enhancement of $54.4 \%$ over traditional ESPRIT when the source separation between $1^{\circ}-4^{\circ}$ among all the previous approaches.

\subsection{Effect of SNR variations}

The effect of SNR variation on proposed modified-ESPRIT and EVM, MUSIC, ESPRIT, PCA is studied and the result obtained is illustrated in Fig. 8. It can be shown that even at high SNR (20 dB), EVM, MUSIC, ESPRIT, and PCA have RMSE greater than the modified ESPRIT, but when SNR decreased, all estimators exhibits the large value of standard deviation while the modified-ESPRIT has the lower value.

The proposed modified-ESPRIT algorithm achieves enhancement in DOA over traditional ESPRIT by $77.8 \%$ when SNR between $0-10 \mathrm{~dB}$. That means the modified-ESPRIT can estimate DOA with lower RMSE than others at the same SNR as shown in Table 3. 


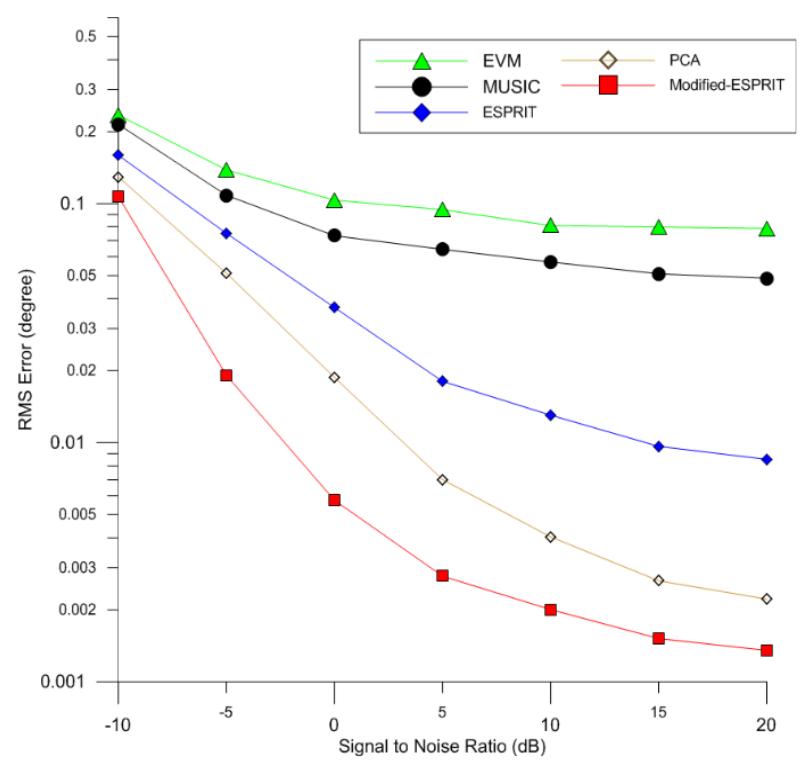

Figure. 8 RMS error vs. SNR for different DOA methods

Table 3. Comparison results of the proposed modifiedESPRIT and different methods in terms of SNR

\begin{tabular}{||l|l|l|l||}
\hline \multirow{2}{*}{ Algorithm } & \multicolumn{3}{|l|}{ Root Mean Square Error $\left(\right.$ deg.) $\times \mathbf{1 0}^{-1}$} \\
\cline { 2 - 4 } & $\begin{array}{l}\text { Less } \\
\text { than 0 } \\
(\mathbf{d B})\end{array}$ & $\begin{array}{l}\text { Between 0 - } \\
\mathbf{1 0} \\
(\mathbf{d B})\end{array}$ & $\begin{array}{l}\text { Between 10 - } \\
\mathbf{2 0}(\mathbf{d B})\end{array}$ \\
\hline EVM [28] & 1.85 & 0.92 & 0.79 \\
\hline $\begin{array}{l}\text { MUSIC } \\
{[30]}\end{array}$ & 1.61 & 0.65 & 0.49 \\
\hline $\begin{array}{l}\text { ESPRIT } \\
{[6]}\end{array}$ & 1.17 & 0.22 & 0.09 \\
\hline PCA [23] & 0.91 & 0.10 & 0.03 \\
\hline $\begin{array}{l}\text { Modified- } \\
\text { ESPRIT }\end{array}$ & 0.63 & 0.04 & 0.02 \\
\hline
\end{tabular}

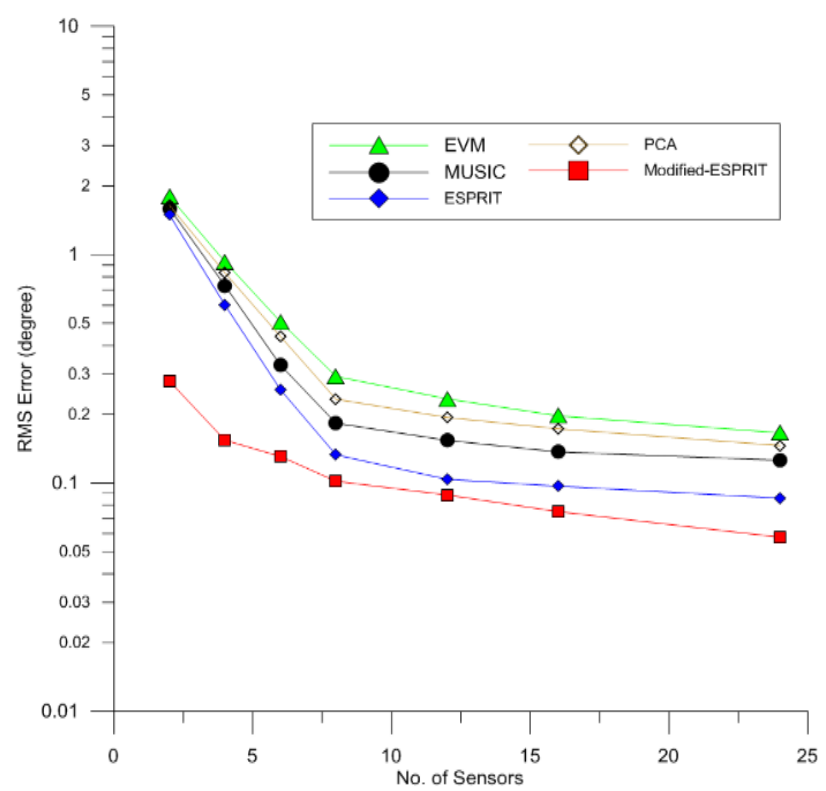

Figure. 9 RMS error vs. source number of sensors for different DOA methods
Table 4. Comparison results of the modified-ESPRIT and different methods in terms of the number of sensors

\begin{tabular}{|l|l|l|l||}
\hline \multirow{2}{*}{ Algorithm } & \multicolumn{3}{|l|}{ Root Mean Square Error (deg.) } \\
\cline { 2 - 4 } & $\begin{array}{l}\text { Less than } \\
\text { 4 sensors }\end{array}$ & $\begin{array}{l}\text { Between 4 - } \\
\text { 12 sensors }\end{array}$ & $\begin{array}{l}\text { More th to 24 } \\
\text { sensors }\end{array}$ \\
\hline EVM [28] & 1.36 & 0.34 & 0.18 \\
\hline $\begin{array}{l}\text { MUSIC } \\
\text { [30] }\end{array}$ & 1.16 & 0.22 & 0.13 \\
\hline ESPRIT [6] & 1.05 & 0.16 & 0.09 \\
\hline PCA [23] & 1.24 & 0.28 & 0.16 \\
\hline $\begin{array}{l}\text { Modified- } \\
\text { ESPRIT }\end{array}$ & 0.22 & 0.10 & 0.07 \\
\hline
\end{tabular}

\subsection{Effect of number of sensors}

From Fig. 9, it is seen that for the number of sensor $\mathrm{M}=24$; the proposed modified-ESPRIT algorithm can estimate DOA with the lowest MSER as compared to the other estimators. As the number of sensors decreased to two, the RMSE increased for all algorithms except the modified ESPRIT still there are lower values of standard deviation.

For the sake of clarity, the RMSE of the proposed modified-ESPRIT, EVM, MUSIC, ESPRIT and PCA algorithms are listed in Table 4, where the number of sensors is varied up to 24 elements, it is clear that an enhancement of $79.04 \%$ is achieved when four or fewer elements are used.

\section{Conclusion}

Estimation of DOA for the propagated signals represents a vital task in large numbers of engineering applications. In this paper, different algorithms of high-resolution signal processing for DOA estimation are examined. ESPRIT is one of these algorithms which have superior resolution properties over the other methods (EVM, MUSIC, and PCA). However, the estimation bias at short separation distances can be considered the main challenge of the ESPRIT algorithm. Hence, a modified-ESPRIT algorithm is proposed and tested The performance evaluation reveals that the proposed modified-ESPRIT algorithm has the best resolution capability than PCA, EVM, MUSIC and the traditional ESPRIT algorithm particularly at low SNR values, small number of snapshots, small number of sensors and very close sources. The proposed modified-ESPRIT can resolve two sources with an enhancement of $83.09 \%$ over traditional ESPRIT when the number of snapshots is very short, while an enhancement of $54.4 \%$ is achieved when source separation between (1 to 4) degrees and $77.8 \%$ achieved when SNR between 0- 
$10 \mathrm{~dB}$ and of $79.04 \%$ when four or fewer elements are used.

Some thoughts can be investigated for further future work such as, extending the study of the behaviour of these algorithms under colored noise, and considering more study on the case where the sources are partially or fully correlated to reach a further understanding of algorithms performance and limitations.

\section{Conflicts of Interest}

The authors declare no conflict of interest.

\section{Author Contributions}

Conceptualization, Azad R. Kareem; methodology, Azad R. Kareem and Ali M. Mahmood; software, Azad R. Kareem; validation, Azad R. Kareem and Ali M. Mahmood; formal analysis, Azad R. Kareem and Ali M. Mahmood; investigation, Azad R. Kareem and Ali M. Mahmood; resources, Azad R. Kareem and Ali M. Mahmood; data curation, Azad R. Kareem; writing original draft preparation, Azad R. Kareem; writing review and editing, Azad R. Kareem and Ali M. Mahmood; visualization, Azad R. Kareem and Ali M. Mahmood; supervision, Azad R. Kareem; project administration, Azad R. Kareem; funding acquisition, not funded.

\section{References}

[1] Z. Meng and W. Zhou, "Direction-of-arrival estimation in coprime array using the ESPRITbased method", Sensors, Vol. 19, No. 3, pp. 707, 2019.

[2] Z. Feng, Z. Fang, Z. Wei, X. Chen, Z. Quan, and D. Ji, "Joint radar and communication: A survey", China Communications, Vol. 17, No. 1, pp. 1-27, 2020.

[3] Z. Kassas, J. Khalife, K. Shamaei, and J. Morales, "I hear, therefore I know where I am: Compensating for GNSS limitations with cellular signals", IEEE Signal Processing Magazine, Vol. 34, No. 5, pp. 111-124, 2017.

[4] M. Boddapati and P. Kishore, "Bandwidth Enhancement of CPW-Fed Elliptical Curved Antenna with Square SRR", International Journal of Intelligent Engineering and Systems, Vol. 11, No. 2, pp. 68-75, 2018.

[5] R. Schmidt, "Multiple emitter location and signal parameter estimation", IEEE Transactions on Antennas and Propagation, Vol. 34, No. 3, pp. 276-280, 1986.
[6] R. Roy and T. Kailath, "ESPRIT-estimation of signal parameters via rotational invariance techniques", IEEE Transactions on Acoustics, Speech, and Signal Processing, Vol. 37, No. 7, pp. 984-995, 1989.

[7] A. Herzog and E. Habets, "Eigenbeam-ESPRIT for DOA-vector estimation", IEEE Signal Processing Letters, Vol. 26, No. 4, pp. 572-576, 2019.

[8] W. He, X. Yang, and Y. Wang, "A HighResolution and Low-Complexity DOA Estimation Method with Unfolded Coprime Linear Arrays", Sensors, Vol. 20, No. 1, pp. 218-227, 2020.

[9] S. Wang and X. Zhou, "Direction-of-arrival and frequency estimation in array signal processing", Journal-Shanghai Jiaotong University-Chinese Edition-, Vol. 33, pp. 40-42, 1999.

[10] J. Guan, Z. Hao, W. Chen, Z. Liu, and X. Yu, "Compensation of current transformer saturation based on improved ESPRIT algorithm", In: Proc. of 2016 IEEE 16th International Conference on Environment and Electrical Engineering, Florence, Italy, pp. 1-4, 2016.

[11] N. Karmous, M. El Hassan, and F. Choubeni, "An Improved Esprit Algorithm for DOA Estimation of Coherent Signals", In: Proc. of 2018 International Conference on Smart Communications and Networking, Yasmine Hammamet, Tunisia, pp. 1-4, 2018.

[12] R. Du, J. Wang, and F. Liu, "Unitary-ESPRIT algorithm for carrier frequency offset estimation for interleaved OFDMA uplink systems", Wireless personal communications, Vol. 69, No. 4, pp. 1615-1627, 2013.

[13] A. Serbes and K. Qaraqe, "A Fast Method for Estimating Frequencies of Multiple Sinusoidals", IEEE Signal Processing Letters, Vol. 27, pp. 386-390, 2020.

[14] S. Saponara, M. S. Greco, and F. Gini, "Radaron-Chip/in-Package in Autonomous Driving Vehicles and Intelligent Transport Systems: Opportunities and Challenges", IEEE Signal Processing Magazine, Vol. 36, No. 5, pp. 71-84, 2019.

[15] Q. Fu, B. Jing, and P. He, "Blind DOA Estimation in a Reverberant Environment Based on Hybrid Initialized Multichannel Deep 2-D Convolutional NMF With Feedback Mechanism", IEEE Access, Vol. 7, pp. 179679179689, 2019.

[16] Z. Wei, X. Li, B. Wang, W. Wang, and Q. Liu, "An Efficient Super-Resolution DOA Estimator 
Based on Grid Learning", Radioengineering, Vol. 28, No. 4, pp. 785-792, 2019.

[17] A. Tennant, and B. Chambers, "A two-element time-modulated array with direction-finding properties", IEEE Antennas and Wireless Propagation Letters, Vol. 6, pp. 64-65, 2007.

[18] S. Azzouzi, M. Cremer, U. Dettmar, R. Kronberger, and T. Knie, "New measurement results for the localization of uhf rfid transponders using an angle of arrival (aoa) approach", In: Proc. of IEEE International Conference on RFID, Orlando, USA pp. 91-97, 2011.

[19] A. Rembovsky, A. Ashikhmin, V. Kozmin, and S. Smolskiy, "Direction finding of radio emission sources", Radio Monitoring, Vol. 43, pp. 237-316, 2009.

[20] J. Yin and T. Chen, "Direction-of-arrival estimation using a sparse representation of array covariance vectors", IEEE Transactions on Signal Processing, Vol. 59, No. 9, pp. 44894493, 2011.

[21] K. Srinivas, S. Ganguly, and P. Kishore Kumar, "Performance Comparison of Reconstruction Algorithms in Compressive Sensing Based Single Snapshot DOA Estimation", IETE Journal of Research, pp. 1-9, 2020.

[22] T. Sarkar and X. Yang, "Application of the conjugate gradient and steepest descent for computing the eigenvalues of an operator", Signal Processing, Vol. 17, No. 1, pp. 31-38, 1989.

[23] H. Abdi and L. J. Williams, "Principal component analysis", Wiley Interdisciplinary Reviews: Computational Statistics, Vol. 2, No. 4, pp. 433-459, 2010.

[24] P. Stoica and A. Nehorai, "Study of the statistical performance of the Pisarenko harmonic decomposition method", In: Proc. of Communications, Radar and Signal Processing, Vol. 135, No. 2, pp. 161-168, 1988.

[25] S. Kung, K. Arun, and D. Rao, "State-space and singular-value decomposition-based approximation methods for the harmonic retrieval problem", JOSA, Vol. 73, No. 12, pp. 1799-1811, 1983.

[26] A. Swindlehurst, B. Ottersten, R. Roy, and T. Kailath, "Multiple invariance ESPRIT", IEEE Transactions on Signal Processing, Vol. 40, No. 4, pp. 867-881, 1992.

[27] D. Johnson and S. DeGraaf, "Improving the resolution of bearing in passive sonar arrays by eigenvalue analysis", IEEE Transactions on Acoustics, Speech, and Signal Processing, Vol. 30, No. 4, pp. 638-647, 1982.
[28] S. Visuri, H. Oja, and V. Koivunen, "Subspacebased direction-of-arrival estimation using nonparametric statistics", IEEE Transactions on Signal Processing, Vol. 49, No. 9, pp. 20602073, 2001.

[29] V. Vincent and J. Lei, "Minimax sparse principal subspace estimation in high dimensions", The Annals of Statistics, Vol. 41, No. 6, pp. 179679-179689, 2013.

[30] Y. Choi, "ESPRIT-based coherent source localization with forward and backward vectors", IEEE Transactions on Signal Processing, Vol. 58, No. 12, pp. 6416-6420, 2010.

[31] R. Roy, A. Paulraj, and T. Kailath, "Directionof-arrival estimation by subspace rotation methods-ESPRIT", In: Proc. of ICASSP'86. IEEE International Conference on Acoustics, Speech, and Signal Processing, Tokyo, Japan, Vol. 11, pp. 2495-2498, 1986.

[32] B. Yang, "Projection approximation subspace tracking", IEEE Transactions on Signal processing, Vol. 43, No. 1, pp. 95-107, 1995.

[33] D. Ganage and Y. Ravinder, "DTCWT-ICA based Improved DOA Estimation of Closely Spaced and Coherent Signals with Uniform Linear Array", International Journal of Intelligent Engineering and Systems, Vol. 12, No. 1, pp. 300-309, 2019.

[34] A. Hyvärinen and E. Oja, "A fast fixed-point algorithm for independent component analysis", Neural computation, Vol. 9, No. 7, pp. 14831492, 1997. 